\title{
Differential expression of microRNA in the lungs of rats with pulmonary arterial hypertension
}

\author{
TINGTING XIAO, LIJIAN XIE, MIN HUANG and JIE SHEN
}

Department of Cardiology, Shanghai Children's Hospital, Shanghai Jiao Tong University, Shanghai 200062, P.R. China

Received October 29, 2015; Accepted November 3, 2016

DOI: $10.3892 / \mathrm{mmr} .2016 .6043$

\begin{abstract}
Pulmonary arterial hypertension (PAH) is a chronic disorder of the small pulmonary arteries, and the efficacy of the therapies and the prognosis remain poor. The pathobiology of PAH is complex, and needs to be elucidated by multiple approaches. The present study used a monocrotaline-induced PAH rat model to perform a comprehensive microRNA (miRNA) microarray screening in the lungs and identified 16 downregulated miRNAs in the lungs from PAH rats. High-enrichment gene ontology (GO) analysis identified several sets of genes, and established the miRNA-mRNA network by outlining the interactions of miRNA and GO-associated genes. Three downregulated miRNAs [miRNA 125-3p (miR-125-3p), miR-148-3p and miR-193] displayed the most marked regulatory function, and miR-148-3p and miR-193 were observed to have the highest number of target mRNAs. Signaling pathway analysis demonstrated 26 signal transduction pathways, with MAPK, TGF- $\beta$ and cell cycle signaling as the most prominent. In addition, 342 genes were identified as the potential targets of these 16 miRNAs. Thus, a set of miRNAs in the lungs from rats with PAH and novel associations between biological events and PAH pathogenesis were identified, providing potential therapeutic targets for this disorder.
\end{abstract}

\section{Introduction}

Pulmonary arterial hypertension (PAH) is a chronic disorder of the small pulmonary arteries and vasculature, characterized by increasing vascular narrowing, leading to progressive pulmonary vascular resistance, with potentially fatal consequences, such as right heart failure $(1,2)$. As a multifactorial disorder, PAH can be idiopathic or associated with underlying conditions, including scleroderma, rheumatoid arthritis

Correspondence to: Dr Jie Shen, Department of Cardiology, Shanghai Children's Hospital, Shanghai Jiao Tong University, 355 Lu Ding Road, Shanghai 200062, P.R. China

E-mail: jshen201412@163.com

Key words: microarray, microRNA, expression, pulmonary arterial hypertension and infection (3). The pathobiology of PAH is complex, and involves diverse pathological events, including vasoconstriction, thrombosis, inflammation, structural remodeling of pulmonary vessels, in addition to disordered production of vasoactive mediators $(1,2,4,5)$. Despite an improved understanding of the therapeutic development of PAH, the efficacy of the therapies and prognosis remain unsatisfactory. Therefore, it is essential to screen sensitive and specific therapeutic targets and biomarkers to provide improved clinical tools for early diagnosis and therapeutic evaluation of PAH.

Owning to its analytical abilities and efficient and accurate simultaneous expression of a large volume of genes, microarray technology has been widely implemented in the investigation of complicated disorders, including cancer, cardiovascular diseases and diabetes $(6,7)$. As the pathogenesis of PAH is complex, it is suggested that various genes and environmental factors are involved. The expression of key genes and regulatory networks challenge the traditional methods, while microarray technology provides a potential tool to further explore the pathogenesis of PAH $(8,9)$. Additionally, high-throughput screening aids in the identification of potential biomarkers for diagnosis, prognosis and as therapeutic targets. Several studies have taken advantage of gene microarray to analyze the peripheral blood cells and smooth muscle cells from $\mathrm{PAH}$ patients $(9,10)$, and identified multiple key genes involved in $\mathrm{PAH}$, such as anti-bradykinin B2 receptor, demonstrating the powerful potential of high-throughput technology to aid in the understanding of the pathophysiology of PAH.

miRNAs are small non-coding RNAs that have emerged as key post-transcriptional modulators of gene expression, acting by targeting mRNAs for translational repression or destabilization (11). Hundreds of mammalian miRNAs have been identified, and they are important in various biological events and pathological processes $(12,13)$. For PAH, reduced plasma miR-150 has been demonstrated to be associated with poor survival (14). Although the source and working mechanism of miR-150 remain to be fully elucidated, it appears that it has a pivotal role in the pathogenesis of PAH. An additional study has reported the emerging role of miRNAs during this process; however, miRNAs in PAH remain to be fully investigated (15).

The present study intended to comprehensively analyze the miRNA profile of lung tissues in monocrotaline-induced PAH rat models. The present study identified 16 downregulated miRNAs in smooth muscle cells from PAH rats. Targeted gene prediction and gene ontology (GO) analysis identified certain 
unexpected signaling pathways, which potentially serve as therapeutic targets for PAH.

\section{Materials and methods}

Monocrotaline-induced PAH animal model. A total of 35 adult male Wistar rats (7 rats as controls, 28 rats as PAH models) were purchased from the Shanghai Laboratory Animal Center (Chinese Academy of Sciences, Shanghai, China), weighing 350-400 g. Animals were fed standard chow and administered a subcutaneous injection of monocrotaline (MCT) $60 \mathrm{mg} / \mathrm{kg}$ (MCT group; Sigma-Aldrich; Merck Millipore, Darmstadt, Germany) or $0.9 \%$ saline as the control group (sham; $n=7$ ). The weight and health status were monitored regularly, and the rats were euthanized using 3\% chloral hydrate (47335-U; Sigma-Aldrich; Merck Millipore) to induce anesthesia at days 7, 14, 21 and 28 following MCT administration. The lungs were removed and lavaged before further treatment. All animal experiments were approved by The Institutional Animal Care and Use Committee of Shanghai Jiaotong University (Shanghai, China) and were in accordance with the Guide for the Care and Use of Laboratory Animals (16).

RNA preparation and quality control. Rat lungs were homogenized and total RNA (enriched for small RNA) was extracted according to manufacturer's instructions using the mirVana miRNA isolation kit (Ambion; Thermo Fisher Scientific, Inc., Waltham, MA, USA), and RNA concentration was quantified using NanoDrop ND-2100 (Thermo Fisher Scientific, Inc.). The RNA integrity was assessed using Agilent 2100 (Agilent Technologies, Inc., Santa Clara, CA, USA) according to the manufacturer's instructions. To ensure a robust analysis, total RNA was assessed by electrophoresis and the RNA integrity number (RIN). Only samples with acceptable XVIIIS and 28S peaks and RIN values greater than eight were included for miRNA profile analysis.

miRNA microarray expression profile. Total RNAs from PAH rats and controls were used for miRNA microarray profiling. miRNA expression was determined by Affymetrix miRNA 3.0 array (Affymetrix, Inc., Santa Clara, CA, USA) containing probes for a total of 1,242 rat miRNAs. The sample labeling, microarray hybridization and washing were performed according to the manufacturer's instructions. Briefly, total RNA were tailed with Poly A and then labeled with Biotin, followed by hybridization onto the microarray. After washing and staining (GeneChip Fluidics Station 450; Affymetrix, Inc.), the arrays were processed using the Affymetrix Scanner 3000 (Affymetrix, Inc.). The scanned images were analyzed using Expression Console software (version, 3.1; Affymetrix, Inc.).

Reverse transcription-quantitative polymerase chain reaction $(R T-q P C R)$. Based on the microarray results, candidate miRNAs were selected for further validation with RT-qPCR. TRIzol reagent (Invitrogen; Thermo Fisher Scientific, Inc.) was used to prepare the total RNAs from lung tissue for miRNA quantitation, and the RNA was reverse transcribed using the TaqMan miRNA reverse transcription kit (Applied Biosystems; Thermo Fisher Scientific, Inc.) and random hexamer primer. The 10 ng cDNA was used as template for miRNA-specific stemloop primers (Applied Biosystems; Thermo Fisher Scientific, Inc.) according to the manufacturer's instructions. miRNA expression assays were conducted using TaqMan primers and probes (Applied Biosystems; Thermo Fisher Scientific, Inc.) for endogenous control small RNAs RNU48 (ID 001006) and target miRNAs. Each sample was run in triplicate for analysis. The primers (miRNA-specific stem-loop primers) and miRNA-specific PCR primer/probe mix were based on the miRNA sequences obtained from the miRBase database. Relative quantifications were performed using the Applied Biosystems 7900HT Fast Real-time PCR system. Data were collected using SDS software (version 2.3; Applied Biosystems; Thermo Fisher Scientific, Inc.). Subsequent to being normalized to RNU48, the expression levels of miRNAs were calculated using the $2^{-\Delta \Delta C q}$ method (17). One-way analysis of variance tests were performed for global comparisons and Tukey's post hoc tests for pair-wise comparisons using SPSS software (version 19.0; IBM SPSS, Armonk, NY, USA).

GO analysis. GO analysis was used to perform enrichment analysis of gene sets based on the GO classification database. For this analysis, Fisher's exact test and the $\chi^{2}$ test were used to classify the GO category, and the false discovery rate (FDR) was calculated to correct the P-value, as smaller FDR represents smaller errors in the P-values. P-values for the GOs of all the differentially expressed genes were computed, and the significance of the function was measured based on enrichment analysis.

miRNA target prediction and pathway analysis. The target genes of the differentially expressed miRNAs were predicted with TargetScan software, (version 6.2; http://www.targetscan. org/vert_40/) and miRanda (August 2010; (http://www. microrna.org/microrna/home.do). The search was based on the 3'-UTR regions of target mRNAs with a minimum miRNA seed length of seven. Only the genes identified simultaneously by the two programs were regarded as potential target genes.

To identify the significant pathway of the differentially expressed genes, Kyoto Encyclopedia of Genes and Genomes (KEGG) was used for the pathway enrichment analysis. Similarly, the Fisher's exact test and $\chi^{2}$ test were used to determine the P-values and FDR. Based on differential expression values, the association between the miRNAs and genes was computed, and the miRNA gene network was constructed according to the interactions of miRNA and genes in the Sanger miRNA database.

miRNA-GO-network. The miRNA-GO-network is built according to the association between significant GOs and genes and the associations among miRNAs and genes. In the gene network, circles represent genes and squares represent miRNAs, and their associations are represented by edges. The center of the network is represented by degree, which means the contribution made by one miRNA to the surrounding genes, or the contribution made by one gene to the surrounding miRNAs. The key miRNAs and genes in the network always have the largest degrees.

Statistical analysis. Student's t-test or one way analysis of variance tests were used, unless otherwise specified, to determine 

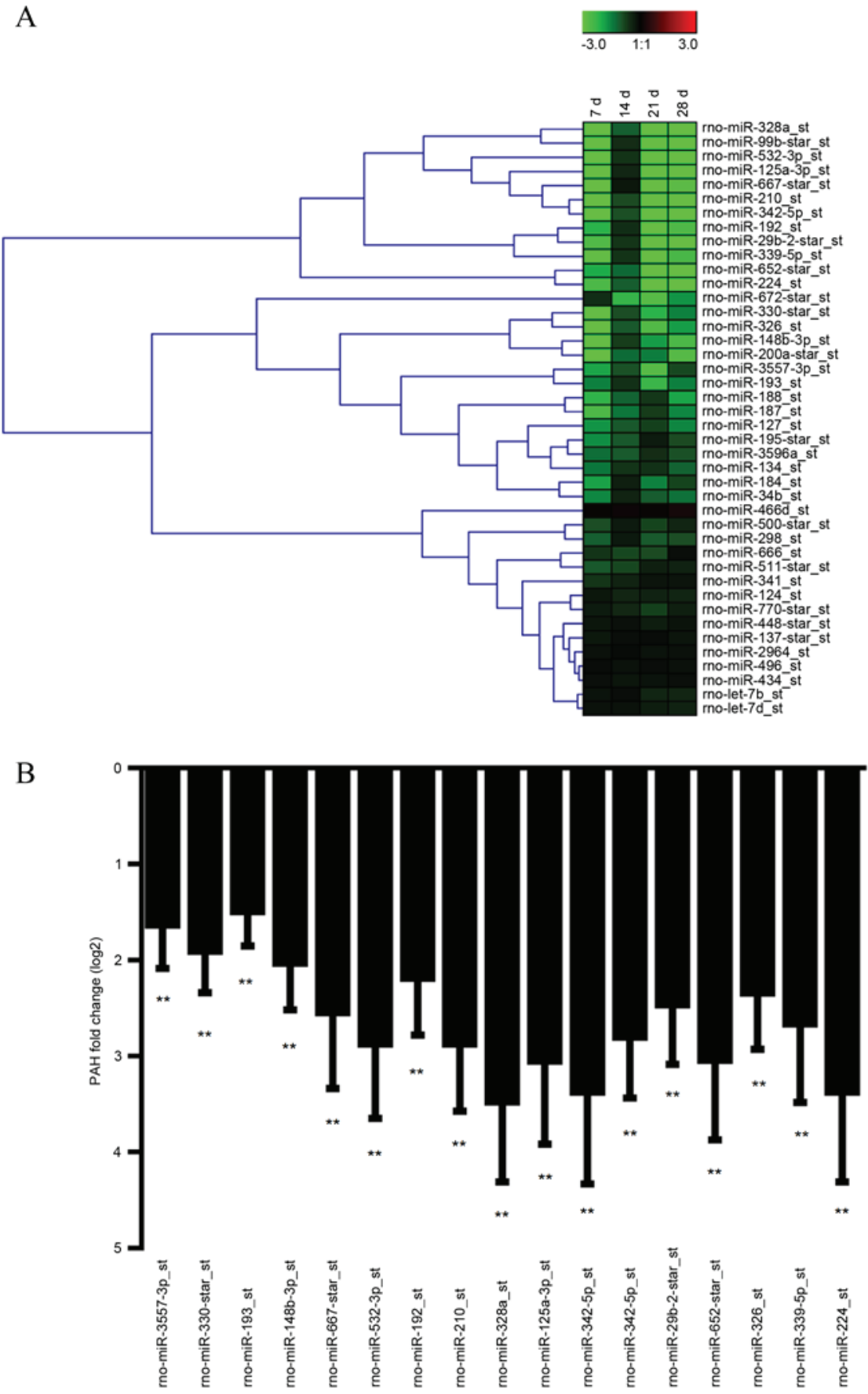

Figure 1. Differential expression of miRNAs between PAH mice and controls. (A) Hierarchical clustering of the differential expression of miRNAs between PAH and control mice. For each miRNA, green represents underexpressed and red overexpressed values compared with the average value (median), in black. Dendrograms (tree graphs) between miRNAs are depicted, where the closest branches of the tree represent miRNAs with the most similar expression pattern. (B) A total 17 miRNAs exhibited significantly difference fold changes $(\log 2)$ in expression of PAH mice when compared with controls ( $\left.{ }^{* *} \mathrm{P}<0.01\right)$. Differential levels are normalized to all assessed miRNAs and relative to control mice. Bars represent the standard error of the mean. miR, microRNA; PAH, pulmonary arterial hypertension.

the significance between PAH and healthy controls using SPSS software (version 19.0; IBM SPSS). P<0.05 was considered to indicate a statistically significant difference.

\section{Results}

miRNA expression profile in lung of $P A H$ rats. Using an Affymetrix miRNA 3.0 array, the study investigated the global expression profile of mature miRNA in lungs from
PAH rats. Total RNAs from PAH rats and controls were used for miRNA microarray profiling. The Affymetrix miRNA 3.0 array contains probes for a total of 1,242 rat miRNAs, and was used to capture the expression signaling of 440 miRNAs. Compared with the control group, 42 miRNA displayed markedly reduced expression (Fig. 1A) in lungs from PAH rats. After normalization, a total of $17 \mathrm{miRNAs}$ exhibited significant expression changes in lungs from $\mathrm{PAH}$ rats $(\mathrm{P}<0.01$; Fig. 1B). Among them, miR-3557-3p is constantly expressed 
Target gene sig go

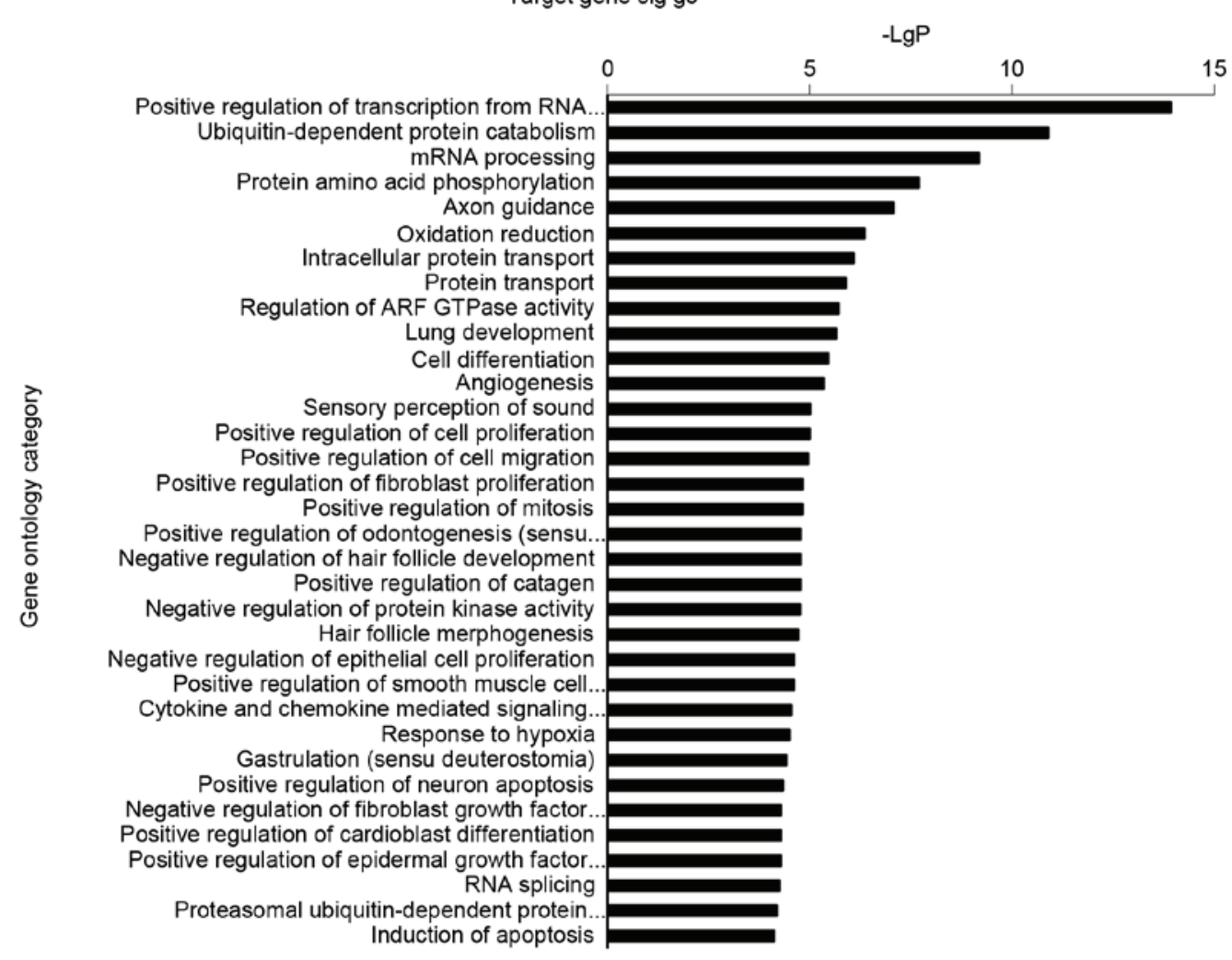

Figure 2. The GO analysis of the differentially expressed miRNAs in lungs from PAH and control rats. The solid bars are the GOs targeted by downregulated miRNA. The vertical axis is the GO category and the horizontal axis is the enrichment of GO. GO, gene ontology; miRNAs, microRNAs; PAH, pulmonary arterial hypertension; ARF, ADP-ribosylation factor.

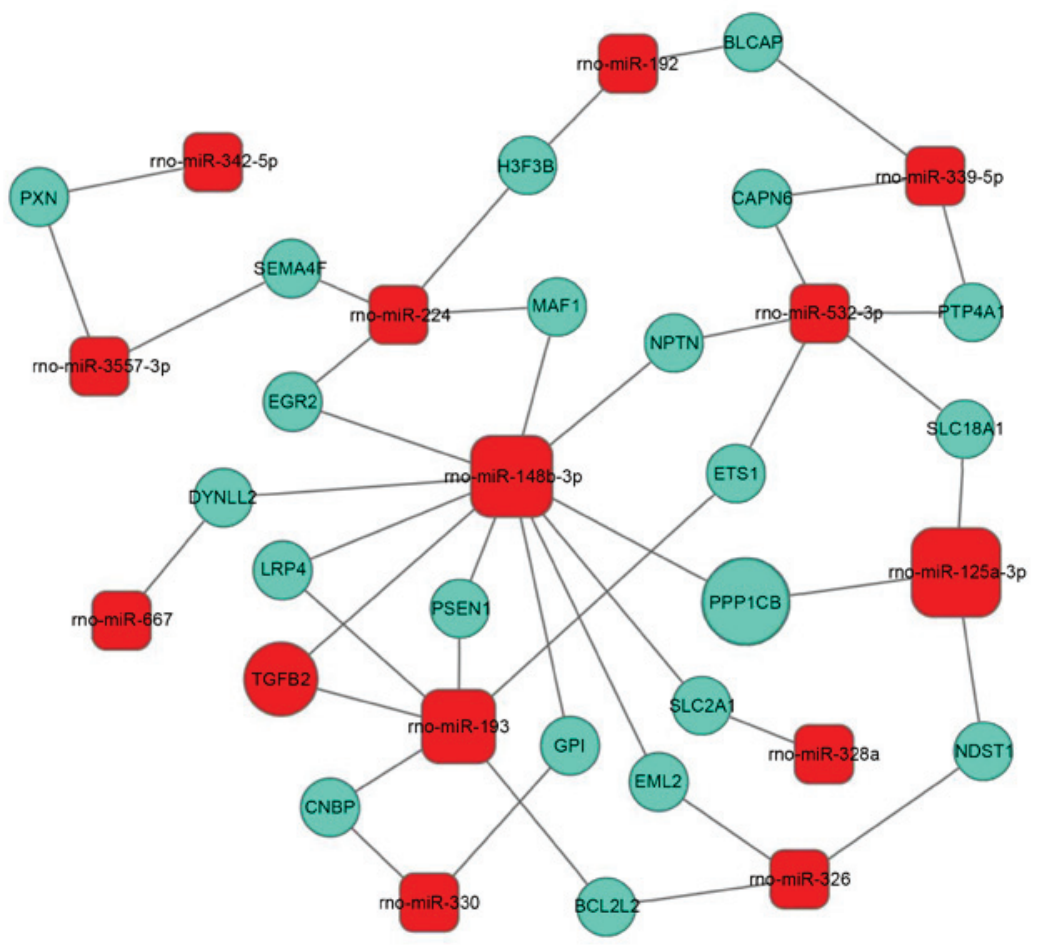

Figure 3. miRNA gene network. Red boxes represent upregulated miRNA and blue boxes downregulated miRNA. Edges represent the inhibitive effect of miRNA on mRNA. miRNAs, microRNAs.

in control animals, while no signaling was detected in PAH rats. In addition RT-qPCR was performed to validate the array results for all 17 miRNAs, and the data showed similar results, confirming the expression change of the miRNAs. 


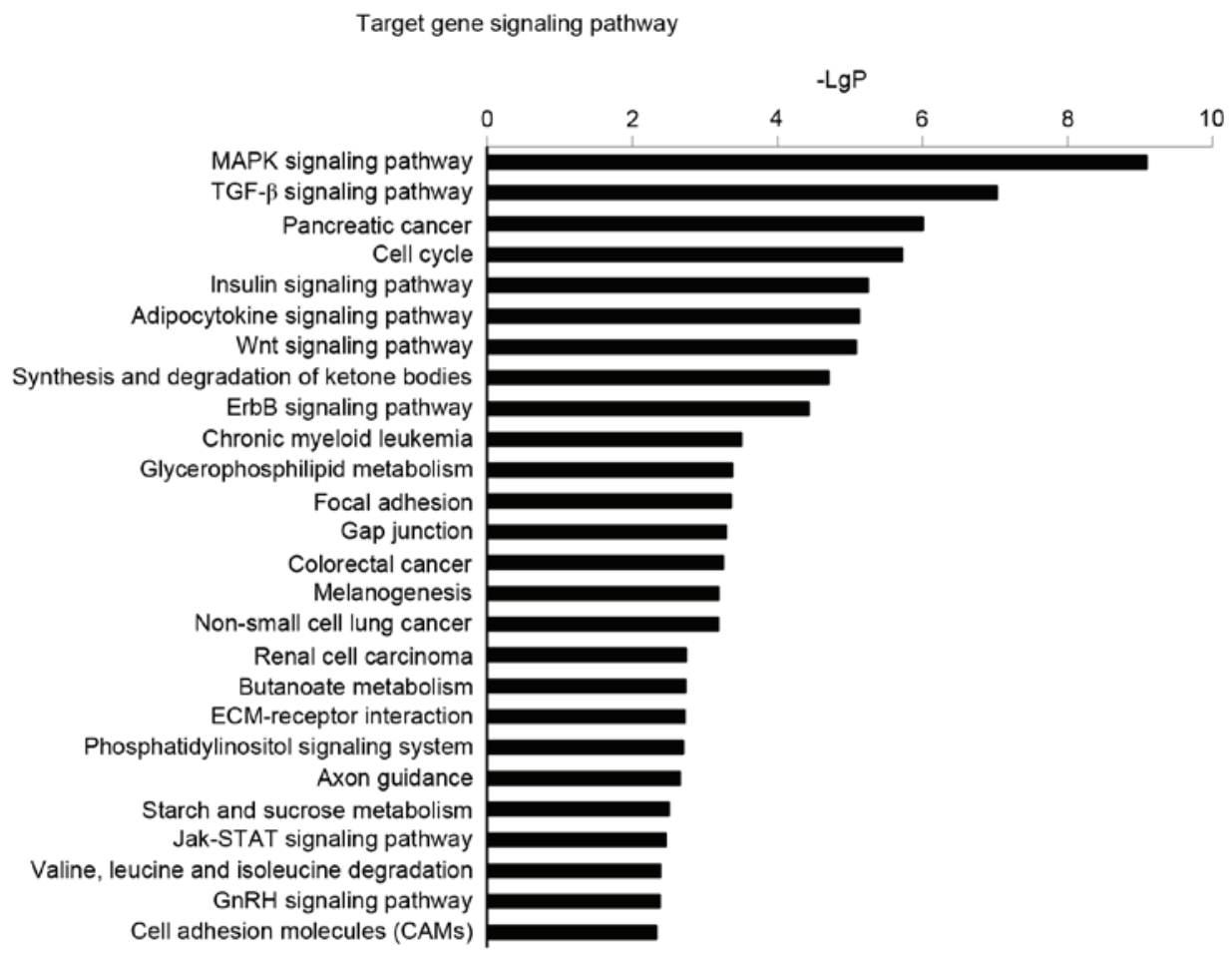

Figure 4. Signaling pathway analysis regulated by miRNA-targeted genes. The vertical axis is the pathway category, and the horizontal axis is the enrichment of pathways. miRNAs, microRNAs; MAPK, mitogen-activated protein kinase; TGF- $\beta$, transforming growth factor $\beta$, Jak, Janus kinase; STAT, signal transducer and activator of transcription; GnRH, gonadotropin-releasing hormone.

Microarray-based $G O$ analysis. $\mathrm{P}<0.05$ was considered the threshold of significance for GOs regulated by miRNAs. In the present study, the highly enriched GOs corresponding to downregulated miRNAs included positive regulation of transcription from RNA polymerase I promoter, ubiquitin-dependent protein catabolism, mRNA processing, protein amino acid phosphorylation, oxidation reduction and intracellular protein transport (Fig. 2). Subsequently, miRNA-mRNA network analysis was performed integrating these miRNAs and GOs by outlining the interactions of miRNA and GO-associated genes (Fig. 3). Three downregulated miRNAs (miR-125-3p, miR-148-3p and miR-193) displayed the most marked regulatory function, and miR-148-3p and miR-193 were demonstrated to target the highest number of mRNAs. Among which, miR-125-3p regulates protein phosphatase 1 catalytic subunit b (PPP1CB), which may bind tumor protein p53 binding protein 2 (TP53BP2) to form a complex to regulate cellular apoptosis (18). miR-148-3p is predicted to regulate transforming growth factor $\beta 2$ (TGF- $\beta 2$ ), which serves multiple functions in diverse biological events, therefore, may be of importance in $\mathrm{PAH}$.

Signaling pathways regulated by differentially expressed miRNA. In order to further elucidate the regulatory function of miRNAs in signal transduction, functional analysis of miRNAs was implemented by KEGG and the results demonstrated that 26 signal transduction pathways were upregulated in PAH (Fig. 4). The prominent upregulated signaling pathways including mitogen-activated protein kinase (MAPK), TGF- $\beta$ and the cell cycle have been demonstrated to participate in the pathogenesis of pulmonary arterial hypertension, suggesting essential roles for these pathways in PAH.
miRNA target analysis. In total, 342 genes were identified with TargetScan and miRanda to be the potential targets of these 15 miRNAs. According to GO analysis and signaling pathway analysis, these genes potentially participate in numerous biological effects, including cell cycle, cell proliferation, differentiation and apoptosis. Of note, axon guidance potentially served an unexpected role in PAH pathogenesis (Fig. 2). Similarly, the pathway analysis also indicated a significant enrichment in axon guidance (Fig. 4), which may possibly lead to the elucidation of a novel mechanism and therapeutic approaches.

\section{Discussion}

Multiple factors, genetic and environmental, participate in the pathogenesis of PAH, which involves a multitude of cellular and molecular elements (19). Proliferation of smooth muscle cells in the small pulmonary arteries is commonly observed in all forms of PAH. Other events, including abnormal proliferation of endothelial cells, inflammation, thrombosis and platelet dysfunction are also common $(1,4,20)$. Given the universal role of miRNA in biological regulation, it is expected that certain miRNAs, including miR-32, 20, 245 and 122 have been reported to be involved in PAH (21). The present study identified certain novel miRNAs by employing a comprehensive miRNA array screening, including miR-125-3p, miR-148-3p and miR-193.

miRNAs exert their functions by regulating target proteins. For example, in PAH the miR-210 expression is decreased, and the downregulation of miR-204 increases expression of SHP2, initiating a signaling cascade involving Src kinase and signal transducer and activator of transcription 3 activation, 
thus promoting pulmonary vessel wall thickening and smooth muscle proliferation (22). As for the newly discovered genes, although no studies have reported an association with PAH, the target prediction provides insight into how these miRNAs are involved in the condition. miR-125-3p is expressed in normal lung tissue, and involved in cellular apoptosis in lung cancer cells. One of the predicted targets of miR-125-3p is PPP1CB, which may bind TP53BP2 to form a complex to regulate cellular apoptosis (18), and is also involved in pulmonary arterial hypertension (9). However, the notable association between miR-125-3p and PAH pathogenesis requires further research.

TGF- $\beta$ has been reported to contribute to PAH development, as endothelial TGF- $\beta$ /activin receptor-like kinase $1 /$ endoglin signaling leads to pulmonary blood vessel angiogenesis, macrophage infiltration, and cytokine expression in the lungs $(23,24)$. The present study identified miR-148-3p in PAH, and identified TGF- $\beta$ as a potential target of miR-148-3p. The association between TGF- $\beta$ and miR-148-3p necessitates further investigation, as a positive answer to this may potentially lead to the development of novel therapeutic approaches. Another promising topic is whether axon guidance is involved in PAH pathogenesis, an area which remains to be investigated fully. In addition, it is suggested that the underlying mechanism may be an association between neuroscience and PAH (3).

The present study identified a set of miRNAs in the lungs from PAH animals and identified unexpected associations between biological events and PAH pathogenesis, which may aid in the development of provide strategic therapeutic approaches. However, limitations were present in the study; for example, no cell-specific identification of these miRNAs was conducted, and how these miRNA function in PAH remains unclear. This should be the subject of further investigations.

\section{Acknowledgements}

The present study was supported by the Shanghai Committee of Science and Technology, China (grant no. 14ZR1434300).

\section{References}

1. Rabinovitch M, Guignabert $\mathrm{C}$, Humbert $\mathrm{M}$ and Nicolls MR Inflammation and immunity in the pathogenesis of pulmonary arterial hypertension. Circ Res 115: 165-175, 2014.

2. Tuder RM, Stacher E, Robinson J, Kumar R and Graham BB Pathology of pulmonary hypertension. Clin Chest Med 34: 639-650, 2013

3. Zamanian RT, Kudelko KT, Sung YK, de Jesus Perez V, Liu J and Spiekerkoetter E: Current clinical management of pulmonary arterial hypertension. Circ Res 115: 131-147, 2014.

4. Nogueira-Ferreira R, Ferreira R and Henriques-Coelho T: Cellular interplay in pulmonary arterial hypertension: Implications for new therapies. Biochim Biophys Acta 1843: 885-893, 2014

5. Perros F, Humbert M and Cohen-Kaminsky S: Pulmonary arterial hypertension: A flavor of autoimmunity. Med Sci (Paris) 29: 607-616, 2013 (In French).

6. Saei AA and Omidi Y: A glance at DNA microarray technology and applications. Bioimpacts 1: 75-86, 2011.
7. Szelinger S, Pearson JV and Craig DW: Microarray-based genome-wide association studies using pooled DNA. Methods Mol Biol 700: 49-60, 2011.

8. Menon S, Fessel J and West J: Microarray studies in pulmonary arterial hypertension. Int J Clin Pract Suppl: 19-28, 2011.

9. Yu J, Wilson J, Taylor L and Polgar P: DNA microarray and signal transduction analysis in pulmonary artery smooth muscle cells from heritable and idiopathic pulmonary arterial hypertension subjects. J Cell Biochem 116: 386-397, 2015.

10. Bull TM, Coldren CD, Moore M, Sotto-Santiago SM, Pham DV, Nana-Sinkam SP, Voelkel NF and Geraci MW: Gene microarray analysis of peripheral blood cells in pulmonary arterial hypertension. Am J Respir Crit Care Med 170: 911-919, 2004.

11. Ha M and Kim VN: Regulation of microRNA biogenesis. Nat Rev Mol Cell Biol 15: 509-524, 2014.

12. Hata A: Functions of MicroRNAs in cardiovascular biology and disease. Annu Rev Physiol 75: 69-93, 2013.

13. O'Connell RM, Rao DS and Baltimore D: microRNA regulation of inflammatory responses. Annu Rev Immunol 30: 295-312, 2012.

14. Rhodes CJ, Wharton J, Boon RA, Roexe T, Tsang H, Wojciak-Stothard B, Chakrabarti A, Howard LS, Gibbs JS, Lawrie A, et al: Reduced microRNA-150 is associated with poor survival in pulmonary arterial hypertension. Am J Respir Crit Care Med 187: 294-302, 2013.

15. Wu D, Talbot CC Jr, Liu Q, Jing ZC, Damico RL, Tuder R, Barnes KC, Hassoun PM and Gao L: Identifying microRNAs targeting Wnt/ $\beta$-catenin pathway in end-stage idiopathic pulmonary arterial hypertension. J Mol Med (Berl) 94: 875-885, 2016.

16. National Research Council (US) Committee on Educational Programs in Laboratory Animal Science. Education and Training in the Care and Use of Laboratory Animals: A Guide for Developing Institutional Programs. National Academies Press, Washington, DC, 1991

17. Livak KJ and Schmittgen TD: Analysis of relative gene expression data using real-time quantitative PCR and the 2(-Delta Delta C(T)) method. Methods 25: 402-408, 2001.

18. Leotta M, Biamonte L, Raimondi L, Ronchetti D, Di Martino MT, Botta C, Leone E, Pitari MR, Neri A, Giordano A, et al: A p53-dependent tumor suppressor network is induced by selective miR-125a-5p inhibition in multiple myeloma cells. J Cell Physiol 229: 2106-2116, 2014.

19. Montani D, Günther S, Dorfmüller P, Perros F, Girerd B, Garcia G, Jaïs X, Savale L, Artaud-Macari E, Price LC, et al: Pulmonary arterial hypertension. Orphanet J Rare Dis 8: 97, 2013.

20. Huertas A, Perros F, Tu L, Cohen-Kaminsky S, Montani D, Dorfmüller P, Guignabert C and Humbert M: Immune dysregulation and endothelial dysfunction in pulmonary arterial hypertension: A complex interplay. Circulation 129: 1332-1340, 2014.

21. Wang Y, Xue XY, Liu YX, Wang KF, Zang XF, Wang J, Wang PL, Zhang J, Pan L, Zhang SY and Wang JX: Pulmonary arterial hypertension and microRNAs-an ever-growing partnership. Arch Med Res 44: 483-487, 2013.

22. Courboulin A, Tremblay VL, Barrier M, Meloche J, Jacob MH, Chapolard M, Bisserier M, Paulin R, Lambert C, Provencher S and Bonnet S: Kruppel-like factor 5 contributes to pulmonary artery smooth muscle proliferation and resistance to apoptosis in human pulmonary arterial hypertension. Respir Res 12: 128, 2011.

23. Ferreira Rde C, Montenegro SM, Domingues AL, Bandeira AP, Silveira CA, Leite LA, Pereira Cde A, Fernandes IM, Mertens AB and Almeida MO: TGF beta and IL13 in Schistosomiasis mansoni associated pulmonary arterial hypertension; a descriptive study with comparative groups. BMC Infect Dis 14: 282, 2014.

24. Gore B, Izikki M, Mercier O, Dewachter L, Fadel E, Humbert M, Dartevelle P, Simonneau G, Naeije R, Lebrin F and Eddahibi S: Key role of the endothelial TGF-b/ALK1/endoglin signaling pathway in humans and rodents pulmonary hypertension. PLoS One 9: e100310, 2014. 